\title{
Un continente diviso
}

\author{
Silvio Pons
}

\section{Stalin and the Fate of Europe: The Postwar Struggle for Sovereignty \\ by Norman Naimark}

The Belknap Press, 368 pp., \$29.95.

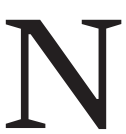

ORMAN NAIMARK è uno dei più autorevoli studiosi della politica di Stalin nelle origini della guerra fredda in Europa. Il suo ultimo lavoro deve essere visto come il compimento di molti anni di ricerca e come un contributo destinato per molti anni a costituire un punto di riferimento fondamentale per gli storici. Egli ha selezionato sette casi di studio che mostrano la complessità delle strategie geopolitiche di Stalin dopo la Seconda guerra mondiale, il loro carattere variabile e talvolta contraddittorio ma mai univoco. I casi di studio riguardano l'isola danese di Bornholm, l'Albania, la Finlandia, l'Italia del 1948, il blocco sovietico di Berlino del 1948-49, la Polonia tra la fine della guerra e il 1949, l'Austria fino al trattato del 1955. La scelta dell'autore si rivela molto felice perché non riguarda esclusivamente la sfera d'influenza sovietica e permette di ricostruire le diverse strategie di Mosca senza cedere alla tentazione di generalizzazioni spesso adottate dagli studiosi. Il ruolo degli attori locali appare spesso influente e persino decisivo, molto più di quanto si sia generalmente ritenuto, e dimostra come la storia dell'Europa posbellica possa essere compresa soltanto tramite l'interazione tra le grandi potenze e i soggetti politici e sociali nel contesto domestico delle nazioni. Una simile visione è stata sinora avanzata soltanto in relazione all'Europa occidentale ${ }^{1}$. L'autore suggerisce che essa vada adottata per l'intero continente, o per gran parte di esso, anche in molti luoghi dove giunse l'influenza sovietica. "Against the backdrop of the war», osserva Naimark, «the new leaders of Europe shared a commitment to the sovereignty and independence of their countries as well as to the rebuilding of their nation's economies and societies». Alcuni di essi vedevano il futuro dei propri paesi inestricabilmente legato alle potenze dominanti, ma erano casi isolati. Invece,

all, even the vast majority of the communists who had entered politics during the anti-German partisan move- ment or in the campaign for the "new democracies" after the war, were committed to ensuring their country's ability to make its own decisions about the future, despite the constraints foisted upon them by foreign occupation forces ${ }^{2}$.

L'Europa postbellica, afferma Naimark, «was no place for utopian fantasies». Le sofferenze, le privazioni, i lutti e le distruzioni provocate dalla guerra in tutto il continente, ma soprattutto nella sua parte centro-orientale, la violenza di massa su base etnica e razziale, le deportazioni e le vittime tra i civili, e infine le vendette dell'ultimo anno di guerra, avevano lasciato un segno profondo. La ricostruzione dell'Europa non poteva che svolgersi nel nome della democrazia e dell'antifascismo, ma non nel nome di rivoluzioni e radicalismi. Nei primi anni del dopoguerra, le tensioni tra i vincitori della guerra si manifestarono nella forma di legittimi, sebbene spesso confliggenti, interessi di sicurezza. La Realpolitik staliniana si inserì in questo contesto politico e psicologico. Perciò, sostiene l'autore, le sorti dell'Europa erano più fluide e aperte di quanto non abbia sostenuto gran parte della storiografia ${ }^{3}$.

Nell'aprile 1945, Stalin dispensò al comunista jugoslavo Milovan Đilas la propria visione dell'Europa postbellica, dichiarando che ciascuno dei vincitori avrebbe imposto «il proprio sistema sociale» fin dove fossero arrivati i rispettivi eserciti. Rese famose dalle memorie di Đilas all'inizio degli anni Sessanta, queste parole di Stalin hanno catturato l'immaginazione e le narrazioni degli storici, in quanto confermavano lo sguardo retrospettivo sulla guerra fredda in Europa. Tuttavia, una scrupolosa analisi del dopoguerra mostra che le cose non dovevano andare necessariamente così, almeno per un certo periodo di tempo. La presenza dell'Armata Rossa non portò all'instaurazione del sistema sovietico in Austria o in Finlandia e neppure in Cecoslovacchia, da dove i sovietici si ritirarono lasciando che si svolgessero libere elezioni (che registrarono una clamorosa affermazione del partito comunista, l'unico evento del genere nell'Europa centro-orientale). Le mosse differenziate, e talvolta contraddittorie di Stalin interagirono con l'azione di attori locali tutt'altro che passivi.

Naimark propone una visione delle politiche di Stalin che aveva già avanzato in passato, corroborandola ora 
con un'evidenza storica molto articolata ${ }^{4}$. Egli respinge la narrazione, diffusa nella letteratura, che Stalin avesse un piano immediato di sovietizzazione dell'Europa e che il suo obiettivo fosse dividere in due il continente. A suo giudizio, Stalin era più sensibile al problema della sicurezza dell'Unione sovietica e guardava soprattutto al futuro della Germania e della Polonia. La sua principale preoccupazione era evitare una ripresa del nazionalismo tedesco e rovesciare il cordon sanitaire nell'Europa orientale in una fascia di sicurezza dell'Unione Sovietica, esercitando una piena influenza su Varsavia, Helsinki e Bucarest. Egli era più interessato a disegnare sfere d'influenza favorevoli a Mosca che non a fomentare rivoluzioni nell'Europa occidentale e meridionale. È probabile che guardasse al futuro del socialismo come un processo graduale e basato su esperienze di economie miste e democrazie antifasciste, non soltanto nell'Europa orientale ma anche in paesi come l'Italia, la Grecia e persino la Gran Bretagna. In vari casi, anche se non sempre, le sue ripetute affermazioni fatte tra il 1944 e il 1946 circa lo scenario di un «nuovo tipo di democrazia» dovrebbero essere prese sul serio, specie quelle rivolte ai comunisti polacchi.

Naimark traccia una precisa periodizzazione del dopoguerra europeo. La reazione sovietica alla «dottrina Truman» e al lancio del Piano Marshall tra il febbraio e il giugno 1947 portò alla creazione del Cominform nel settembre 1947, un organismo internazionale che raggruppava i principali partiti comunisti europei. Fu questo il momento della svolta. La tesi dei «due campi» inconciliabili nella politica mondiale, enunciata da Andrei Zhdanov, rappresentò un'autentica dichiarazione di guerra fredda $\mathrm{e}$ delineò la divisione dell'Europa. Il colpo di Stato comunista a Praga nel febbraio 1948 e il blocco di Berlino dal giugno 1948 al maggio 1949 generarono una sfera di dominio esclusivo, che deprivava della loro sovranità i paesi dell'Europa centro-orientale. Nello stesso tempo, questi avvenimenti ebbero forti ripercussioni politiche e psicologiche nell'Europa occidentale, che portarono alla instaurazione della NATO. In altre parole, una catena di eventi e reazioni alimentò le sindromi ideologiche e di sicurezza presenti nella cultura politica sovietica, produsse l'esportazione del modello staliniano e interagì con la costruzione del blocco occidentale. Prima che quella spirale divenisse irreversibile, tuttavia, molti leader politici, comunisti e anticomunisti, manifestarono e condivisero in modi diversi l'obiettivo della sovranità e dell'indipendenza dei rispettivi paesi, anche sull'onda della partecipazione popolare alle elezioni democratiche.

Alcuni dei casi di studio ricostruiti dall'autore sono già stati oggetto di analisi storica, come è ovvio, ma acquistano di significato e mostrano implicazioni ancora più rilevanti nel quadro delle interconnessioni e sincronie evidenziato dal volume, che non si limita all'esperienza della sovietizzazione. In particolare, è interessante notare le coincidenze temporali e, in una certa misura, persino l'interazione tra diversi eventi e scenari nella fase finale di passaggio da un'Europa soggetta a «sfere d'influenza» ben delineate, ma non ancora esclusive, a un'Europa divisa in blocchi politici e militari, vale a dire l'anno 1948. La sorte della Finlandia, ad esempio, è stato spesso considerata nella sua singolarità, mentre ci appare qui in una luce diversa, cioè non soltanto come l'unico paese non sovietizzato sotto l'influenza sovietica, ma come un caso di compromesso raggiunto tramite l'interazione tra soggetti locali e grandi potenze. Una dinamica in atto anche altrove, sebbene con risultati diversi. La neutralità filo-sovietica finlandese fu largamente il risultato della strategia di un leader come Juho Kusti Paasikivi per garantire la sovranità del proprio paese, emarginando i comunisti nella politica interna. Stalin ritenne che un simile compromesso fosse più che accettabile, accontentandosi di limitare l'autonomia del paese nella politica estera. Nell'aprile 1948, malgrado timori o attese diffusi, non si verificò a Helsinki alcun colpo di Stato sul modello di quello praghese ${ }^{5}$. Il colpo di Praga costituì una minaccia ancora più forte, almeno sotto il profilo psicologico, in Austria. Tuttavia, malgrado la presenza militare sovietica, anche in questo caso una opzione del genere non fu davvero avanzata. Le politiche di Stalin rivelarono indecisione e oscillazioni. L'influenza dei socialdemocratici e del loro leader Karl Renner e l'evidente impopolarità dei sovietici e dei comunisti aprirono la strada a una diversa soluzione e al lungo negoziato per un trattato, destinato a concludersi soltanto dopo la morte di Stalin.

Le coeve elezioni italiane dell'aprile 1948 si collocano in un diverso contesto strategico, ma rivelano dinamiche di interazione analoghe sebbene più complesse, data la presenza degli Stati Uniti. Nel libro, l'Italia è l'unico caso di studio della politica di Stalin verso un paese incluso nella sfera occidentale, anche se le prospettive di una neutralità italiana non erano del tutto escluse in quel momento. Il caso italiano appare particolarmente rilevante perché le circostanze della guerra ne fecero presto una specie di laboratorio delle relazioni tra gli alleati in vista del dopoguerra. Dopo lo sbarco degli angloamericani in Sicilia e la caduta del regime fascista il 25 luglio del 1943, il paese fu diviso in due parti dal momento dell'armistizio dell'8 settembre in avanti. Lo Stato italiano si sfaldò drammaticamente. Nel Nord invaso dall'esercito tedesco si insediò una Repubblica neo-fascista guidata da Mussolini e nacque un forte movimento armato di Resistenza antifascista, nel Sud liberato dagli eserciti angloamericani si formò il governo del Maresciallo Badoglio sotto la monarchia. Marginalizzata dal regime armistiziale sotto il controllo occidentale, l'Unione Sovietica reagì riconoscendo il governo Badoglio nel marzo 1944. La mossa di Stalin consentì a Togliatti di realizzare la famosa «svolta di Salerno», con la dichiarazione che i comunisti erano disposti a collaborare unitariamente con il governo e proponevano di rinviare la scelta tra repubblica e monarchia alla fine della guerra. 
Così la partita tra le grandi potenze favorì la nascita di un partito comunista di massa legittimato sul piano istituzionale nella sfera d'influenza occidentale.

Il realismo di Togliatti e del suo gruppo dirigente evitò che la grave situazione sociale del paese sboccasse in una rivoluzione alla fine della guerra. Tuttavia, la nascita della Repubblica in Italia e l'elezione di un'Assemblea Costituente, nel giugno 1946, si svolse in un contesto conflittuale destinato a conoscere una escalation con lo sviluppo della guerra fredda. Il lancio del Piano Marshall coincise con la fine dei governi di coalizione antifascista, decisa dal leader del partito di maggioranza, il cattolico Alcide De Gasperi, nel maggio 1947. Dal quel momento in avanti, la tensione tra le forze nazionali salì alle stelle, riflettendo in pieno la divisione bipolare internazionale. I leader politici italiani furono abbastanza saggi da rispettare l'Assemblea Costituente e consentire l'approvazione di una Costituzione democratica e antifascista. Ma il rischio di una guerra civile italiana analoga a quella greca fu percepito da tutti gli attori in campo, in vista delle decisive elezioni politiche dell'aprile 1948. La creazione del Cominform spinse i comunisti occidentali a mobilitarsi contro il piano di aiuti americano, fomentando scioperi che potevano costituire il prologo di un'insurrezione o comunque provocare reazioni repressive massicce.

La mobilitazione di massa e la reciproca propaganda resero incandescente la vigilia delle elezioni. Tutti gli attori nazionali e internazionali si interrogavano non soltanto sull'esito ma anche sul rischio di un conflitto fuori controllo. George Kennan disegnò lo scenario di una guerra civile e di una divisione militare dell'Italia per prevenire una vittoria del Fronte popolare, l'ambasciatore James Dunn immaginò un conflitto armato scatenato dai comunisti nel caso di una loro sconfitta. I comunisti italiani paventavano un colpo di Stato reazionario sostenuto da Washington nel caso di una loro affermazione elettorale. I cattolici usarono toni apocalittici temendo a loro volta una presa del potere comunista sul modello di Praga. Da entrambe le parti si apprestarono strutture paramilitari, anche se non è molto chiara la loro effettiva consistenza e influenza. Il risultato elettorale fu nettamente a favore delle forze moderate e anticomuniste. La Democrazia Cristiana giunse vicina alla maggioranza assoluta dei voti, mentre il Fronte Popolare subì una cocente sconfitta. Un simile esito, che non si prestava a sospetti di brogli e sotterfugi, contribuì a placare gli animi.

$\mathrm{Fu}$ fondamentale la funzione moderatrice dei principali leader politici italiani, che usarono toni calibrati e si adoperarono per evitare lo scontro. Il leader comunista, in particolare, si consigliò con Mosca circa l'opportunità o meno di una scelta insurrezionista, che molti nel suo partito evocavano più o meno esplicitamente e che avrebbe contato sul sostegno di Tito e della Jugoslavia. Togliatti non si limitò però a chiedere il parere di Stalin, ma usò argomenti volti a scongiurare per quanto possi- bile una catastrofe. Affermò infatti che una guerra civile italiana avrebbe inevitabilmente avuto incontrollabili conseguenze internazionali e portato a una nuova guerra mondiale. Dichiarò anche che egli avrebbe fatto la scelta ultima della sollevazione soltanto in circostanze estreme, indipendentemente dal parere dei partner sovietici. Stalin approvò la linea di Togliatti e lo sconsigliò anche di seguire i suggerimenti degli jugoslavi.

Questa scelta realista venne mantenuta anche poco più tardi, al momento di un evento potenzialmente dirompente come l'attentato a Togliatti del 14 luglio 1948. Il leader comunista fu colpito con tre colpi di pistola all'uscita dal Parlamento, per mano di uno studente di simpatie fasciste suggestionato dalla violenza del discorso pubblico anticomunista. Consapevoli della lezione appresa nei mesi precedenti, i dirigenti del partito promulgarono uno sciopero generale, ma non incoraggiarono la spontanea mobilitazione popolare che avrebbe potuto sfuggire al controllo e che soltanto in casi limitati sboccò in forme di violenza. Lo stesso Togliatti invitò alla calma dal suo letto d'ospedale. Ancora una volta Mosca mandò segnali di approvazione per il realismo dei comunisti italiani. La scomunica appena pronunciata contro Tito e i suoi compagni poteva soltanto accentuare la scelta realista in Italia. Le elezioni italiane furono un test significativo per la costituzione del blocco occidentale e Washington le recepì come l'inizio di una nuova fase per contrastare l'influenza comunista nella guerra fredda. Ma esse mostrano anche il self-containment dei comunisti locali. De Gasperi fu l'indiscusso vincitore della contesa e le sue scelte determinarono le sorti dell'Italia per molto tempo a venire, anche in quanto egli fu l'artefice dell'ingresso del paese nella NATO l'anno successivo. Anche Togliatti però, nota Naimark, può essere considerato con un leader «pursuing Italian sovereignty and building Italian democracy, despite his unquestionable loyalty to Moscow» ${ }^{6}$.

Il blocco di Berlino si verificò poco dopo le elezioni italiane, in una catena di eventi destinata a produrre la creazione dei blocchi. L'autore analizza un aspetto molto trascurato dagli storici, vale a dire il ruolo che ebbe il blocco nel cambiare le politiche sovietiche in Germania, sollecitando la reazione dei soggetti locali. L'identità stessa dei berlinesi occidentali si forgiò in quella congiuntura. L'evidenza degli archivi mostra che Stalin decise il blocco per affermare gli interessi sovietici in Germania e che non lo concepì come il possibile prologo di un conflitto militare. Egli si proponeva di impedire la formazione di uno Stato tedesco occidentale. La sua condotta e strategia resta però opaca, perché la tensione prodotta dal blocco non favorì affatto un simile obiettivo, tanto più che i contatti diplomatici vennero sospesi per un anno circa. Secondo Naimark, alla fine della guerra Stalin era contrario all'idea di dividere la Germania tra est e ovest, anche perché la parte orientale del paese era più piccola e debole nelle sue capacità industriali. Egli 
avrebbe preferito una Germania unita e neutrale, non sovietizzata ma sottoposta in larga misura all'influenza di Mosca sul modello della Finlandia.

Stalin ritenne probabilmente che una posizione attendista avrebbe raggiunto questo risultato. Non si aspettava la capacità occidentale di accettare una Germania economicamente in ripresa, specie da parte dei britannici e dei francesi, che si manifestò già al momento del lancio del Piano Marshall. Il blocco rappresentò una reazione e un tentativo di indebolire la cooperazione occidentale, che continuava a puntare sull'illusione di poter dividere tra loro gli alleati occidentali, ottenendo l'esito opposto. «Instead of dividing the West», nota Naimark, «the blockade contributed to the building of the Atlantic Alliance» ${ }^{7}$. Stalin seguì due politiche contraddittorie tra loro. Da una parte, spinse i comunisti tedeschi sin dalla fine del 1948 a instaurare un governo nella zona di occupazione sovietica, anche se non una «dittatura del proletariato». Dall'altra parte, continuò a insistere sull'idea di una Germania unita ancora nel 1949, alla vigilia della nascita dello Stato tedesco occidentale.

Ma le stesse mosse di Mosca avevano alimentato la nascita di un attore locale imprevisto e destinato ad avere una storia cruciale nei decenni successivi, i berlinesi occidentali. Il discorso pronunciato dal leader socialdemocratico (ed ex comunista) Ernst Reuter il 9 settembre 1948 "was one of the most dramatic - and effective - of the early Cold War period», con il suo appello al mondo intero di guardare alla città di Berlino e alla sua popolazione come difensori delle libertà di tutti ${ }^{8}$. Il sentimento che Reuter veicolò fu determinante per impedire che la costruzione dello Stato tedesco occidentale si svolgesse separatamente dal destino di Berlino Ovest e anzi potesse persino presupporre di abbandonare la città nella zona sovietica. Reuter influenzò sensibilmente le scelte del Generale Clay e dell'Amministrazione americana garantendo la resistenza dei berlinesi e la possibilità di successo del ponte aereo, che infine vanificò la strategia di Stalin.

Una strategia della sovranità nell'ambito del movimento comunista era a dir poco difficile da realizzare in Polonia, ma Wladislaw Gomulka la portò avanti fino a quando le circostanze internazionali la resero impraticabile e il suo stesso artefice finì per essere perseguitato. Anche in questo caso, l'anno 1948 costituì il momento di svolta. Sin dal 1945, Gomulka non esitò ad avanzare critiche e rimostranze a Mosca per la condotta vendicativa dell'Armata Rossa in Polonia, che rendeva i comunisti ancora più impopolari di quanto già non fosse facile prevedere alla luce della storia del paese tra le due guerre. In particolare, l'occupazione sovietica subito dopo il Patto tra Stalin e Hitler, e le conseguenti violenze e deportazioni nel periodo 1939-41, avevano lasciato un segno nella coscienza nazionale polacca che non era stato cancellato. La questione principale era però costituita dalla costruzione di una «democrazia popolare» credibilmente diversa dal modello sovietico. In un paese a maggioranza rurale come la Polonia, ciò significava evitare la collettivizzazione delle campagne, almeno per un lungo periodo di tempo. Stalin e Gomulka raggiunsero un'intesa su questo punto cruciale. La «via polacca al socialismo» non sarebbe stata democratica, dato l'obiettivo condiviso di stabilire un monopolio di fatto del potere da parte del partito comunista, ma neppure una «dittatura del proletariato», che lo stesso Stalin affermò nel maggio $1946^{9}$.

Gomulka mise in atto una simile politica nel dopoguerra, insistendo sull'esigenza di acquisire un profilo nazionale, anche a dispetto dell'opposizione di hardliners quali Berman e Bierut. La creazione del Cominform nel settembre 1947 costituì però un'inversione di tendenza e un'ipoteca ideologica sulla relativa autonomia politica del partito polacco. Anche se nel mirino del Cominform furono inizialmente i comunisti occidentali, Gomulka si rese conto perfettamente che il compromesso postbellico sulla sovranità della Polonia era in pericolo. Probabilmente egli ritenne anche di essere stato ingannato dai sovietici, come affermò più tardi, che presentarono la conferenza dei partiti comunisti europei come un passaggio assai meno impegnativo di quanto doveva essere. Per questo motivo, egli evitò si porsi in prima linea dopo aver ospitato la conferenza fondativa del Cominform, ancor più dinanzi alla rottura tra Stalin e Tito. Seebbene gli jugoslavi fossero protagonisti di una visione intransigente e poco incline al realismo delle «democrazie popolari», la loro condanna annunciava infatti un giro di vite nella relazioni tra Mosca e tutti i paesi dell'Europa centro-orientale. Tra i primi obiettivi del giro di vite figurava proprio Gomulka. Un dossier contro il suo «deviazionismo» nazionalista era già pronto a Mosca nell'aprile 1948, prima ancora che la seconda Conferenza del Cominform espellesse gli jugoslavi. Qualche mese più tardi, nel settembre 1948, fu posto sotto accusa per questo motivo al plenum del Comitato centrale.

Ciò nonostante, Gomulka continuò a difendere la propria linea. Lo fece con tutti i mezzi possibili, anche i più spregiudicati e infamanti. In una strategia di resistenza alle pressioni di Stalin, egli si presentò come il leader che garantiva la causa socialista e patriottica polacca contro l'influenza degli «ebrei» e il loro «nichilismo nazionale». Questo argomento solleticava il crescente antisemitismo di Stalin, che emergeva dalla campagna contro il «cosmopolitismo»e, nello stesso tempo, alludeva all'inaffidabilità e impopolarità di gran parte degli altri leader comunisti polacchi. Ciò non fu sufficiente. Alla fine del 1948, Gomulka entrò in rotta di collisione con Stalin. Prima accettò con riluttanza la proposta del leader sovietico di far parte del nuovo gruppo dirigente del Partito polacco unito dei lavoratori, insieme a Bierut, Berman e Minc. Ma poi, gli scrisse una lettera declinando la richiesta, ancora una volta con argomenti antisemiti. Contribuì così a decretare la propria disgrazia. La vicenda di Gomulka si intrecciò con le epurazioni e i processi in vari paesi dell'Europa sovietizzata dal 1949 in avanti. Egli fu arrestato nell'agosto 1951 e rimase in carcere senza subire un processo fino alla fine del 1954, ben oltre la morte di Stalin. In altre parole, 
il caso della Polonia non registrò alcun compromesso, a differenza della Finlandia. In definitiva, però, osserva Naimark, «Gomulka can be seen as having won his struggle with Stalin, over what he saw as the related issues of the Jews in the Polish Party and the distinct Polish road to socialism $»^{10}$, dal momento che fu richiamato al potere al momento delle fatali crisi sociali e politiche del 1956.

L'unico rimprovero che si può muovere all'autore è di non avere arricchito ulteriormente il suo lavoro con altri casi di studio. Per esempio, una ricostruzione del caso della Cecoslovacchia, unico paese della sfera d'influenza sovietica a mantenere un sistema politico pluralista fino al 1948, sarebbe stata utile per illuminare ancora di più le dinamiche di affermazione e perdita della sovranità. L'influenza paneuropea del colpo di Stato a Praga nel febbraio 1948 fu così rilevante anche per questo motivo. Tuttavia, il libro di Naimark è un'opera già molto ricca e ha il merito di restituirci la complessità di un passato che tendiamo a semplificare. Nelle sue conclusioni, egli può giustamente sostenere di aver mostrato come «the coming of the Cold War unquestionably influenced the fate of Europe in the immediate postwar years, but it was not as dominant a factor as many historians suggest», principalmente perché «the agency of Europeans mattered and mattered a lot». Persino nella sfera sovietica, dove gli europei centro-orientali furono alla fine deprivati della propria libertà e facoltà di scelta, «the struggle was real, and there was little that was inevitable about the division of the continent in the immediate postwar period $»^{11}$. Questa lezione, come l'autore fa notare, non è priva di significati per la memoria degli europei, perché in definitiva essi vinsero la lotta per la sovranità tramite l'integrazione e la distensione, fino al collasso dei regimi comunisti e la nascita dell'Unione Europea.
Silvio Pons è professore di Storia contemporanea alla Scuola Normale Superiore di Pisa.

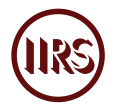

1. Si veda, ad esempio, Federico Romero, Storia della guerra fredda: L'ultimo conflitto per l'Europa (Turin: Einaudi, 2009).

2. Norman Naimark, Stalin and the Fate of Europe: The Postwar Struggle for Sovereignty (Cambridge: The Belknap Press of Harvard University Press, 2019), 21.

3. Per una narrazione convenzionale, si veda Anne Applebaum, Iron Curtain: The Crushing of Eastern Europe, 1944-1956 (New York: Knopf Doubleday, 2013). Per una ricostruzione che abbraccia un arco cronologico più ampio, fornendo una lettura della sovietizzazione come prodotto inevitabile dell'incontro tra l'Unione Sovietica staliniana e l'Europa, cfr. Timothy Snyder and Ray Brandon, eds., Stalin and Europe: Imitation and Domination (Oxford \& New York: Oxford University Press, 2014).

4. Norman Naimark, The Russians in Germany: A History of the Soviet Zone of Occupation, 1945-1949 (New Haven: Harvard University Press, 1995).

5. Naimark, Stalin and the Fate of Europe, 119.

6. Naimark, Stalin and the Fate of Europe, 268.

7. Naimark, Stalin and the Fate of Europe, 193.

8. Naimark, Stalin and the Fate of Europe, 186.

9. Naimark, Stalin and the Fate of Europe, 205.

10. Naimark, Stalin and the Fate of Europe, 229.

11. Naimark, Stalin and the Fate of Europe, 270-72.

DOI: $10.37282 / 991819.21 .32$ 